\title{
Simulating Vascular Systems in Arbitrary Anatomies
}

\author{
Dominik Szczerba and Gábor Székely \\ Computer Vision Lab, ETH, CH-8092 Zürich, Switzerland
}

\begin{abstract}
Better physiological understanding of principles regulating vascular formation and growth is mandatory to their efficient modeling for the purpose of physiologically oriented medical applications like training simulation or preoperative planning. We have already reported on the implementation of a visually oriented modeling framework allowing to study various physiological aspects of the vascular systems on a macroscopic scale. In this work we describe our progress in this field including (i) extension of the presented model to three dimensions, (ii) addition of established mathematical approaches to modeling angiogenesis and (iii) embedding the structures in arbitrary anatomical elements represented by finite element meshes.
\end{abstract}

\section{Introduction}

As pointed out in our previous work [1], the vascular systems do not simply influence organ appearance as part of their surface texture, but also behave like physical objects with certain mechanical properties. In particular, they will deform along with the hosting tissue and lead to bleeding when cut through. The ultimate goal is to provide a tool which, given a 3D representation of a given tissue/organ and an intuitive set of physiologically meaningful parameters, will generate vascular structures in an arbitrary anatomical region. Such systems are not expected to carry only geometrical information but also provide data on mechanical properties of the vascular system and the related blood flow.

In the previous work we have proposed a macroscopic model allowing to generate various vascular systems with high graphical fidelity for simulation purposes. The presented model included the formation of a primitive capillary plexus prior to maturation of the vascular system and treated its later development as a dynamic growth controlled by biophysical factors. This way the remodeling of the vascular system could be described, and full information on biophysical properties and hemodynamic conditions in the system could be provided at any time. The model, successful in generating a diversity of visually appealing vascular structures suffers, however, from a fundamental limitation. Whereas various geometrical constraints on the growth process can be imposed a priori, the domains to be vascularized are basically addressed as continuum and represented analytically. Such treatment is very convenient and widely used in developing and investigating mathematical modeling methods. Their implementation for reallife applications over complex domains represented in discrete forms is, however, not straightforward and needs special attention. The goals of the present work therefore are: 
- the full extension of the existing framework to three dimensions;

- the integration of the established mathematical modeling of angiogenesis with our previous simulation framework with the emphasis on visually realistic appearance;

- the embedding of such vascular networks in real-life anatomical objects defined by finite element meshes.

In practice, these objectives will be strongly inter-dependent. In order to solve differential equations governing the vessel formation one needs to provide a discretization scheme and proceed by finding the solution numerically. This is to be performed on a mesh, with the simplest one to use being an isotropic Cartesian grid. Offering significant implementational simplicity, which is advantageous from the algorithmic point of view, such meshes do not, however, provide the desired flexibility to efficiently map complex anatomical domains. Because of irregularity and high unpredictability, modeling such a diverse range of anatomical geometries is only possible through unstructured meshes. Such meshes are also convenient for modeling elastic properties of the tissue and organs.

Vascular structures are subject to computer modeling for a longer time now. A detailed overview of the available literature is given in our previous work [1] and will not be repeated here. The approaches discussed there include fractal self-similar constructs, functional macroscopic pipelines and continuous mathematical models. Still another group of approaches, not discussed previously, is based on optimization principles of theoretical physiology, which is well exemplified by the recent work by M. Georg et.al. [2]. Their model is initialized by a simple sub-optimal tree filling of the entire organ at a given resolution. It then becomes subject to optimization, mainly intra-vascular volume minimization, which is one of the major principles driving vascular system development as discussed in the literature. As compared to another popular optimization-based method, namely Constrained Constructive Optimization (CCO) [3], this new algorithm can also implement topological changes, which are essential to the optimization process. The generated structures presented by the authors prove to be similar to real experimental data acquired from corrosion casts, with the emerging symmetry explained in terms of global optimality. The fundamental difference to our approach is in that (1) we explicitly address capillary networks supplied by higher order vessels and (2) we model blood vessel formation by relying exclusively on local interactions, without enforcing any global optima. Our approach is well justified in cases of malignant tumors, where the vascular systems are known to be often unstable, leaky or otherwise sub-optimal.

One of the inspirations to continue our previous work is a recent proposition of a non-lattice mathematical model of angiogenesis [4]. Whereas established mathematical models to date restrict endothelial cell movements to 4 discrete directions on an isotropic Cartesian grid, the authors of that work propose to depart from a fixed lattice and adopt the governing equations for arbitrary (continuous) directions. They still, however, use very simple geometrical domains (squares) and provide biochemical agents only in form of analytically prescribed profiles. As described in the next section, we will adopt their topological freedom to model endothelial cell motility but with significant differences in the discretization scheme and representation of the underlying biochemical factors. 


\section{The Model}

The formation of blood vessels during angiogenesis in general - healthy or cancerous is a process where capillary sprouts depart from pre-existing parent vessels in response to externally supplied chemical stimuli. By means of endothelial cell proliferation and migration the sprouts then organize themselves into a branched, connected network structure. In this work we tend to focus specifically on tumor induced angiogenesis, which involve cancerous solid tumor cells secreting a number of biochemicals collectively known as growth factors. The general formalism can, however, also be applied to healthy anatomies if global functional optimality is not the primary focus of investigation. This is the case e.g. in early vascular development stages with only preliminary capillary beds present. Moreover, the remodeling procedure introduced in our previous work can readily be applied to the structures generated here. The preliminary capillary plexus does not necessarily require blood flow to form. Once the blood enters the freshly formed vessels it inflates the elastic walls. Regions of high stress disproportions, where the capillaries started to sprout from the parent vessels are remodeled first. Inflating vessels will not only change their initial distribution of radii but also adjust the bifurcation angles. The optimal bifurcation design discussed so often in the literature may arise from local conditions determined by e.g. the elastic properties of the walls forming the initial tripod. In a completely different modeling approach, involving cells as opposed to pipes used here, we have demonstrated that local variations in the shear stress rebuild a uniform distributions of tissue pillars into a set of non-symmetric bifurcations [5] observed in vivo. Even thought the resulting structure is not guaranteed to be optimal, the initial symmetry is broken and the bifurcations and micro-vessels emerge as a result of a simulation instead of being composed a priori out of basic structural elements as pipes or tripod. Therefore, instead of presumptively imposing optimality rules on the sprouting and bifurcating capillaries we start our modeling from the widely accepted assumption that the initial response of the endothelial cells to the angiogenic growth factors is due to chemotaxis, enforcing the cell migration towards the tumor or ischemic cell. Once secreted, the growth factors diffuse into the surrounding tissue and the extracellular matrix, establishing a certain concentration gradient between the chemical source and the parent vessels. As the endothelial cells migrate through the extracellular matrix in response to this gradient there is some uptake and binding of the growth factors by the cells [6]. Therefore, it can be modeled by a diffusion equation with a natural decay term:

$$
\frac{\partial c}{\partial t}=S_{c}+D_{i} \nabla^{2} c-\theta_{0} c
$$

with $c$ being the chemical concentration, $S_{c}$ its source, $D$ the diffusion coefficient and $\theta$ decay rate. Once the initial distribution of the growth agents due to secretion by the malignant or ischemic cells has been established, endothelial cells start to respond to the stimulus by sprouting, eventually modifying the initial growth factor concentration by cellular bindings. Partial differential equations governing the endothelial density evolution are derived from a general form of transport equation and can be written in the following form: 


$$
\begin{array}{r}
\frac{\partial n}{\partial t}=D_{0} \nabla \cdot\left(f_{0}(c) \nabla n\right)-D_{c} \nabla \cdot\left(f_{c}(c) n \nabla c\right) \\
\frac{\partial c}{\partial t}=-\theta_{1} n c
\end{array}
$$

with $n$ the endothelial cell density and $D_{i}, \theta_{i}$ positive constants.

A similar set of partial differential equations with respect to cellular density evolution was first postulated by [7]. Below is the interpretation of these equations needed for their adaptation to our simulation framework, as well as discussion of the main difference to our modeling approach. The first term in Equation 2, chemokinesis, is the random displacement of the endothelial cells with the transition probability modulated by the concentration of the chemical stimuli. In a specific case of constant growth factor concentration this reduces to the classical diffusion equation with the diffusion constant $D_{0}$, in a general case of non-constant concentrations, however, this term allows to selectively change random motility of the migrating cells. In particular, it is reasonable to assume that as the cells approach the angiogenic source they will become more motile and more chaotic in their migration. In our model we address this issue taking a simple linear form of $f_{0}$. The second term in the same equation is known in literature as chemotaxis. It is the advective term in the general transport equation with the difference in that the advection velocity is replaced by another vector field, namely the gradient of the (scalar) growth factor concentration. This is well justified from experimental observations, as it is widely known that the endothelial cells tend to "climb up" the gradients of biochemical growth stimuli [8]. Similar to Anderson and Chaplain, we take the chemotactic amplitude $f_{c}(c)$ as a receptor-kinetic function of the form $f_{c}(c) \sim 1 / c$. This represents an intuitive assumption of decreased cellular sensitivity with the increased chemical stimulus. The second equation (Equation 3) models the biochemical growth factors concentration by linear uptake functions due to degradation through cellular bindings. We make two important simplifications in our modeling, namely that the concentration of the growth factor does not change throughout the vessel growth process (i.e. Equation 3 is not solved) and that the haptotactic response due to fibronectin, present in the original formulation, is neglected. Inclusion of these components in the transport equation appears to be crucial to obtain physiologically normalizable models, in the first implementation, however, with the emphasis on real-life modeling of the underlying arbitrary hosting tissue, we did not find them mandatory for providing a realistic network coverage of the domain of interest. This could, however, be integrated in the next version of the framework to increase the physiological correctness of the model.

Anderson and Chaplain proceed by discretizing the partial differential equations on an isotropic Cartesian grid and either modeling the endothelial cell density (the continuous model) or by introducing transition probabilities and displacing the endothelial sprouting tips explicitly (the discrete model). Instead, we follow the way sketched for simple geometries in [4] and depart from fixed vascular topology, which allows us to flexibly handle arbitrary finite element meshes.

A detailed description of an example method to generate unstructured tetrahedral meshes out of surfaces (extracted by e.g. segmentation of magnetic resonance imaging of real anatomical objects) can be found in [9]. Here we proceed by interpreting the 
governing equations in terms of a given mesh. We start by discretizing the $\nabla$ operator needed for establishing the initial chemotactic gradient as well as for diffusive transport of the growth factors. In case of tetrahedral meshes a natural choice of discretization is the finite volume scheme. The transport phenomena described by Equation 1 and Equation 2 in such a nomenclature take the following general form:

$$
\int_{V}\left(\frac{\partial \phi}{\partial t}\right) d V+D_{c} \oint_{A} f_{c}(c) n \vec{n} \cdot \nabla c d A=D_{0} \oint_{A} f_{0}(c) \vec{n} \cdot \nabla n d A+\int_{V} S_{c} d V .
$$

with $\phi$ a general scalar field, $V$ denoting the control volume, $A$ control volume surface, $\boldsymbol{n}$ the surface normal vectors (not to be confused with $n$ scalar) and $S_{c}$ the growth factor sources (e.g. secretion by tumor or ischemic cells). We linearize these equations and express their discretized versions using the control volume central values in order to facilitate the iterative solution procedure:

$$
C_{i} \phi_{i}+C_{i, j} \phi_{i, j}=S_{i}^{\phi},
$$

with the Einstein summation over the control element's direct $(i)$ and indirect $(i, j)$ neighbors. $\phi_{i}, \phi_{i, j}$ correspond to the scalar field's cell centered values, and $S^{\phi}$ is determined by the boundary conditions. Derivation of the coefficient matrix $C$ is nontrivial and will not be presented here.

By proceeding with the Eulerian formulation used so far, we would have to solve this kind of equations three times: to establish the initial concentration gradients $(\phi=c)$, and to solve the transport equation terms $(\phi=n$ and $\phi=c)$. Instead, we solve it only once, for the initial steady-state equilibrium of $c$ (Equation 1), and change our formulation of Equation 2 to Lagrangian dynamics of sprouting vessel tips. Such a continuous approach, as opposed to a discrete Eulerian treatment, is particularly beneficial in case of finite element meshes, as the generated vascular structures are limited by neither the underlying mesh resolution nor the topology. In addition, the resulting structure can straightforwardly be converted to a flow network required to solve the Hagen-Poiseuille's flow equations, as described in our previous paper [1]. Therefore, by realizing that the diffusive term in the transport equation is responsible for random motility of the endothelial cells (modulated by $f_{0}$ ), and that the advective term effectively displaces the cells towards the regions of higher chemical concentration, we arrive at the following equation of a sprouting tip motion:

$$
\frac{d \vec{r}}{d t} \equiv \overrightarrow{v_{t o t}}=d_{1}(c) \overrightarrow{v_{0}}+d_{2}(c) \overrightarrow{v_{c}}+d_{3}(c) \overrightarrow{v_{i}},
$$

with $d \boldsymbol{r}$ being the net displacement of the sprouting tip due to the following contributions:

- diffusive motility $\overrightarrow{v_{0}}$ in a random direction (e.g. picking a random point on a sphere),

- directed motility $\overrightarrow{v_{c}}$ in the direction of a local growth factor's gradient (estimated e.g. using the Gauss theorem),

- inertial motility $\overrightarrow{v_{i}}$ enforcing a certain resistance of the sprouting tip to rapidly change the currently followed direction. 
The functions $d_{i}(c)$ regulate the relative importance of the three contributions to the net displacement and depend on the local growth factor concentrations in the same way as the previously discussed coefficients $D_{i}$. We additionally introduce a function $\beta(c)$ regulating bifurcation probability at the sprouting tip and allow for fusion of colliding vessels (anastomosis). In addition, once a vessel reaches a minimal diameter (corresponding to a capillary), it is no more bifurcating (as it would lead to further decrease in diameter, which is not observed in reality). As stated in section 1 - and opposed to our previous approach - we do not anymore attempt to provide optimal bifurcation angles nor diameters a priori, instead we simply chose a random deviation from the followed direction within $[0, \pi)$. Even if such a selection may seem arbitrary, these angles are strongly influenced by chemotaxis, and practically anyway limited to forward directions.

\section{Results}

The model described in the preceding sections was tested with a finite element volumetric mesh of an early development stage of a uterine polyp model presented in [10]. A few interior elements in the structure's head were picked and marked as actively dividing cells, which will be continuously secreting angiogenic growth factors and regulating the endothelial response. Because there is an uptake of these chemicals in the extracellular matrix, as discussed before, a steady state concentration gradient will form after some iterations. Now the vessels are allowed to start sprouting, with the bifurcation probability and random motility increasing and the chemotactic sensitivity decreasing with the concentration of the growth factor. Such separation between the initial and the growth phase is not unrealistic since it is very likely that the sprouting begins only when a certain triggering threshold in the local concentration is reached. The input surface mesh along with the output vascular network, with the initial growth starting from the base of the polyp, is shown in Figure 1. The steady state distribution of the growth factors is visualized on the surface (leftmost) and inside (middle) of the model. Note the chaotic nature of the resulting vessels (right) as compared to the results presented in the
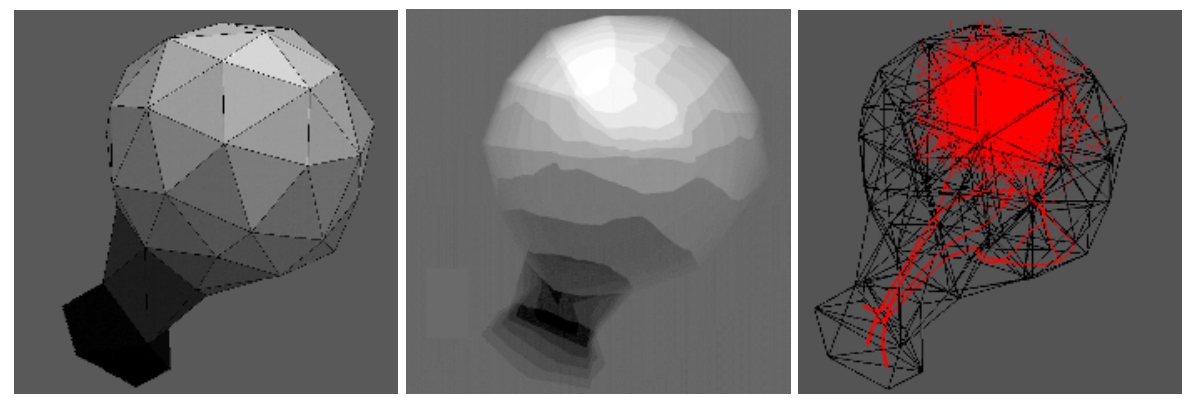

Fig. 1. Simulation results of the vascular growth model described in the text. Left: input mesh with the surface triangles color-coded according to the growth factor concentration on the surface. Middle: volumetric "see-through" visualization of the growth factor concentration inside the model. Right: generated vascular structure and wire-frame of the input mesh. 
previous work [1]. As expected - and as observed in real tumors - the blood vessels become gradually very chaotic as they approach the cancerous epicenter. This is achieved in the simulation by letting the random motility and the bifurcation probability increase rapidly as the tumor is approached. At the same time the vessel tip inertia as well as the ability to reorient itself along the local gradient is continuously quenched. This results in approximately parallel vessels along the polyp's neck and ends with a dense "brush" in the epicenter. The full procedure leading to the results presented above, including mesh generation, establishment of the initial gradients and generation of the vessels, took approximately 10 minutes on a PC with Pentium4/3.0GHz and 1GB RAM. Calculation of flow conditions in the generated network and the according oxygen penetration of the tissue took another 10 minutes.

These results demonstrate the model's ability to deal with realistic conditions encountered in early development stages of small vascular tumors and at the same time providing realistic visualization along with necessary bio-physical information required by surgical training simulations. Moreover, as indicated before, the meshes used for the discretization purposes were designed with the possible tissue elastic deformation in mind. It is straight-forward now to mechanically deform the vessels together with the hosting tissue using e.g. a simple and fast mass-spring modeling. In addition, using the flow network model described in our previous work, we can readily provide the flow conditions at any location of interest and use this information for e.g. visualization of bleeding when a surgeon cuts in the tissue during a virtual training session. Together with the real-time elastic deformation this provides reasonably realistic environment for virtual-reality based medical training.

\section{Outlook}

The presented simulation takes into account basic experimental knowledge of the growing process, namely endothelial cell proliferation and migration, and their modulated response to changes in local growth factor concentrations. Achieved results correspond well to both experimental findings and to the established mathematical models of angiogenesis. In addition, the key components of the previously introduced model - the flow network and stress driven remodeling - can readily be applied. The network coverage is, however, provided under the quasi-static assumption of a steady development state of the tumor and the underlying chemical factors. While the mechanical deformation of the resulting structure can be easily applied, further increase of realism can only be achieved by simultaneous simulation of the vascular growth and the tumor development. This would allow to study both the mechanical interplay between developing structures (e.g. deformation due to stretching and strains) as well as their coupled biochemical dependence. Such modeling is already under way and will soon be integrated into the presented framework.

We are aware that in case of healthy anatomies the generated structures may be suboptimal in the sense of a network coverage, building material or diffusive exchange. The enforcement of such an optimum based exclusively on local interactions will require much better understanding of short-range mechanisms acting on the cellular level, but is an attractive vision for the ultimate explanation of the origins of natural self-symmetry and functional optimality. 


\section{References}

[1] Szczerba, D., Székely, G.: Macroscopic modeling of vascular systems. In: Medical Image Computing and Computer-Assisted Intervention - MICCAI 2002. Volume 2489 of Lecture Notes in Computer Science., LNCS Springer (2002) 284-292

[2] Georg, M., Hahn, H., Preusser, T., Peitgen, H.O.: Global constructive optimization of vascular systems. Submitted to IEEE transactions on Medical Imaging (2004)

[3] Schreiner, W., Neumann, M., Neumann, F., Roedler, S., End, A., Buxbaum, P., Muller, M., Spieckermann, P.: The branching angles in computer-generated optimized models of arterial trees. J. Gen. Physiol. 103 (1994) 975-989

[4] Plank, M.J., Sleeman, B.D.: Lattice and non-lattice models of tumour angiogenesis. Bulletin of Mathematical Biology 66(6) (2004) 1785-1819

[5] Szczerba, D., Székely, G.: Computational model of flow-tissue interactions in intussusceptive angiogenesis. Journal of Theoretical Biology 234 (2005) 87-97

[6] Ausprunk, D.H., Folkman, J.: Migration and proliferation of endothelial cells in preformed and newly formed blood vessels during tumor angiogenesis. Microvascular Research (1977)

[7] Anderson, A., Chaplain, M.: Continuous and discrete mathematical models of tumorinduced angiogenesis. Bulletin of Mathematical Biology 60 (1998) 857-899

[8] Terranova, V., DiFlorio, R., Lyall, R., Hic, S., Friesel, R., Maciag, T.: Human endothelial cells are chemotactic to endothelial cell growth factor and heparin. J. Cell Biol. 101 (1985) 2330-2334

[9] Szczerba, D., Székely, G.: A computational model of micro-vascular growth. In: Simulation of Multiphysics Multiscale Systems, ICCS 2005: 5th International Conference on Computational Science, Atlanta. Volume 3516 of Lecture Notes in Computer Science., Springer-Verlag (2005) 17-24

[10] Sierra, R., Bajka, M., Székely, G.: Pathology growth model based on particles. In Ellis, R.E., Peters, T.M., eds.: Procs. of the Sixth International Conference on Medical Image Computing and Computer-Assisted Intervention. Volume 1., Springer (2003) 25-32 